\title{
Class of Charged Fluid Balls in General Relativity
}

\author{
A. Sah, Prakash Chandra \\ Department of Mathematics, M B Govt. P G College, Haldwani, India \\ Email:archanasah7@gmail.com,pcfsouk@gmail.com
}

How to cite this paper: Sah, A. and Chandra, P. (2016) Class of Charged Fluid Balls in General Relativity. International Journal of Astronomy and Astrophysics, 6, 494-511. http://dx.doi.org/10.4236/ijaa.2016.64038

Received: November 21, 2016

Accepted: December 26, 2016

Published: December 29, 2016

Copyright $\odot 2016$ by authors and Scientific Research Publishing Inc. This work is licensed under the Creative Commons Attribution International License (CC BY 4.0).

http://creativecommons.org/licenses/by/4.0/

\begin{abstract}
In the present study, we have obtained a new analytical solution of combined Einstein-Maxwell field equations describing the interior field of a ball having static spherically symmetric isotropic charged fluid within it. The charge and electric field intensity are zero at the center and monotonically increasing towards the boundary of the fluid ball. Besides these, adiabatic index is also increasing towards the boundary and becomes infinite on it. All other physical quantities such as pressure, density, adiabatic speed of sound, charge density, adiabatic index are monotonically decreasing towards the surface. Causality condition is obeyed at the center of ball. In the limiting case of vanishingly small charge, the solution degenerates into Schwarzchild uniform density solution for electrically neutral fluid. The solution joins smoothly to the Reissner-Nordstrom solution over the boundary. We have constructed a neutron star model by assuming the surface density $\rho=2 \times 10^{17} \mathrm{kgm}^{-3}$. The mass of the neutron star comes $1.58 \mathrm{M} \odot$ with radius $14.574 \mathrm{~km}$.
\end{abstract}

\section{Keywords}

Exact Solution, Einstein's Field Equations, Charged Fluid Ball, Compact Star, General Relativity

\section{Introduction}

An analysis of the Reissner-Nordstrom metric shows that a spherically symmetric distribution of charged dust may avoid the catastrophic gravitational collapse, a seemingly unavoidable feature of Schwarzschild's [1] geometry exterior to an electrically neutral fluid sphere of mass bigger than certain critical limit. As in evidence we have Bonnor's model [2] [3] of the equilibrium ball of charged dust in contrast to the OppenheimerSnyder [4] continually contracting ball of electrically neutral dust. Though Bonnors model has been found to the unstable to small radial perturbations and also to a change in the total charge content of the system, it initiates a general interest in the study of the 
implications of Einstein-Maxwell field equations with reference to the general relativistic prediction of gravitational collapse. On the other hand, it is generally considered that a black hole may carry non-zero net charge, apart from its mass and angular momentum. Such an assumption may require the collapse of stellar masses of charged matter. It has been suggested by Shvartsman [5] that on account of interaction between a star and its surroundings, it is possible that stellar systems carrying electric charge may exist in nature. It is therefore not surprising that in recent years the problem of finding nonsingular, physically meaningful solution of Einstein-Maxwell field equations for static ball of charged coherent perfect has received wide attention. The inclusion of charge seems to affect the stability of the system-the stability of Schwarzschild's uniform density sphere increases by the introduction of net surface charge. It has been shown that the stability is more profound if the same amount of charge be distributed uniformally throughout within the sphere.

The search for the exact solutions is of continuous interest to researcher. Buchdahl [6] proposed a famous bound on the mass radius ratio of relativistic fluid spheres which is an important contribution in order to study the stability of the fluid spheres. DelgatyLake [7] studied all the then existing solutions and established that Adler [8], Heintzmann [9], etc. do not satisfy all the well behaved conditions and also pointed out that only nine solutions are well behaved; out of which seven in curvature coordinates (Tolman [10], Finch and Skea [11], Patvardhav and Vaidya [12], Mehra [13], Kuchowicz [14], Matese and Whitman [15], Durgapal's two solutions [16]) and only two solutions (Nariai [17], Goldman [18]) in isotropic coordinates. Ivanov [19] [20] [21] [22] [23], Neeraj Pant [24], Maurya and Gupta [25], Pant et al. [26] [27], Pant and Sah [28] [29], Tewari, Charan and Chandra [30], Sah, Chandra and Charan [31] studied the existing well behaved solutions of Einstein's field equations. Some pioneer work in Relativity is given by Herrera et al. [32]-[37], Tewari and Charan [38] [39] [40] [41]. Nduka [42] [43], Whitman and Burch [44], Tikekar [45], Ivanov [46], Ray et al. [47], Stettner [48], Krori and Barua [49], Ray and Das [50], Pant and Negi [51], Florides [52], Dionysiou [53], Pant et al. [54] etc. gave the well behaved solution for charged fluid sphere. Pant et al. [55], Pant and Tewari [56], Fuloria et al. [57] gave charge analogue of Heintzmann, Adler, Durgapal's relativistic exact solution respectively. Gupta and Maurya [58] gave charge analogue of Durgapal and Fuloria superdense star. Bijalwan and Gupta [59], Gupta and Kumar [60] gave charge analogue of Schwarzschild's interior solution.

In this paper, we present a new solution of Einstein-Maxwell field equations in spherically symmetric coordinates which are well behaved solutions charge analogous solution of Sah and Chandra [61]. In our present study the paper consists of nine sections. In Section 2, Einstein's field equations for charged fluid sphere in canonical coordinates are given. In Section 3, gravitational binding energy of a charged fluid sphere is given. Section 4 consists of boundary conditions for well behaved solutions. New class of solution of Einstein's field equations for a charged fluid sphere in canonical coordinates is given in Section 5. Section 6 stipulates the properties of this new class of solution of Einstein-Maxwell field equations. In Section 7 the matching conditions of interior 
metric of the charged fluid with the exterior metric are given. For better illustration of our physically accepted solution, the relevant physical quantities are presented by tables and figures in Section 8. Finally, some concluding remarks have been made in Section 9.

\section{Field Equations for a Charged Fluid Sphere in Canonical Coordinates}

The Einstein-Maxwell field equations in general relativity are given by

$$
R_{v}^{\mu}-\frac{1}{2} R g_{v}^{\mu}=-\frac{8 \pi G}{c^{4}} T_{v}^{\mu}
$$

where $R_{v}^{\mu}, R, g_{v}^{\mu}$ and $T_{v}^{\mu}$, are Ricci mixed tensor, scalar curvature, metric tensor and the energy momentum tensor for fluid sphere respectively. $T_{v}^{\mu}$ is conserved quantity such that

$$
T_{v ; \mu}^{\mu}=0
$$

The energy momentum tensor for a charged fluid sphere is defined as

$$
T_{v}^{\mu}=M_{v}^{\mu}+E_{v}^{\mu}
$$

Here $M_{v}^{\mu}$ is the part of the energy momentum tensor due to matter distribution of the system and, for a perfect fluid distribution, it is given by

$$
M_{v}^{\mu}=\left(\rho c^{2}+p\right) u^{\mu} u_{v}-p g_{v}^{\mu}
$$

where $\rho$ and $p$ are the density and isotropic pressure of the fluid element measured locally in its proper reference frame. The density $\rho$ gives total matter energy in proper volume $V$ as

$$
c^{2} M_{p}=\int_{V} \rho c^{2} \mathrm{~d} V
$$

$u^{\mu}$ is element's time-like four-velocity vector such that

$$
g_{v}^{\mu} u^{\mu} u^{v}=1
$$

$E_{v}^{\mu}$ is the part of energy momentum tensor due to electromagnetic character of matter within the fluid sphere and is defined by

$$
E_{v}^{\mu}=\frac{1}{4 \pi}\left[-F^{\mu \lambda} F_{v \lambda}+\frac{1}{4} \delta_{v}^{\mu} F^{\lambda t} F_{\lambda t}\right]
$$

where the electromagnetic tensor $F^{\mu \nu}$ satisfies Mexwells equations

$$
\begin{gathered}
F_{\mu v, \lambda}+F_{v \lambda, \mu}+F_{\lambda v, \mu}=0 \\
\frac{\partial}{\partial x^{v}}\left\{(-g)^{\frac{1}{2}} F^{\mu \nu}\right\}=4 \pi(-g)^{\frac{1}{2}} J^{\mu}
\end{gathered}
$$

$J^{\mu}$ is the 4-current density for a fluid of null charge conductivity and a conserved quantity such that

$$
J_{; \nu}^{\mu}=0
$$

and is given by 


$$
J^{\mu}=\sigma u^{\mu}
$$

$\sigma$ being the charge density of the fluid element and gives the total charge contained in proper volume $V$ as

$$
Q=\int_{V} \sigma \mathrm{d} V
$$

The total non gravitational energy in a proper volume $V$ is given by

$$
E_{p}=\int_{V} T_{0}^{0} \mathrm{~d} V
$$

We consider a static spherically symmetric charged perfect fluid distribution. The interior space-time metric for spherically symmetric fluid distribution in canonical coordinate is given by

$$
\mathrm{d} s^{2}=A^{2} \mathrm{~d} t^{2}-B^{2} \mathrm{~d} r^{2}-r^{2}\left(\mathrm{~d} \theta^{2}+\sin ^{2} \theta \mathrm{d} \phi^{2}\right)
$$

where $A$ and $B$ are functions of $r$ only.

The electrostatic field is described by the only non-singular components $F^{10}$, $F^{01}\left(=-F^{10}\right)$ of $F^{\mu v}$.

In view of (8), (9) and (10) we obtain

$$
F^{01}=\frac{Q c}{r^{2} A B}
$$

where $Q$ stands for the total charge contained within the sphere of radius $r$ and is given as

$$
Q=\int_{0}^{r} 4 \pi r^{2} B \sigma \mathrm{d} r
$$

In view of the metric (14) and energy momentum tensor (3), the field Equation (1) gives

$$
\begin{gathered}
\frac{8 \pi G}{c^{4}} p=\frac{1}{B^{2}}\left(\frac{2 A^{\prime}}{r A}+\frac{1}{r^{2}}\right)-\frac{1}{r^{2}}+\frac{G Q^{2}}{c^{4} r^{4}} \\
\frac{8 \pi G}{c^{4}} p=\frac{1}{B^{2}}\left(\frac{A^{\prime \prime}}{A}+\frac{A^{\prime}}{r A}-\frac{B^{\prime}}{r B}-\frac{A^{\prime} B^{\prime}}{A B}\right)-\frac{G Q^{2}}{c^{4} r^{4}} \\
\frac{8 \pi G}{c^{2}} \rho=\frac{1}{B^{2}}\left(\frac{2 B^{\prime}}{r B}-\frac{1}{r^{2}}\right)+\frac{1}{r^{2}}-\frac{G Q^{2}}{c^{4} r^{4}}
\end{gathered}
$$

In view of Equation (17) and Equation (18), pressure isotropy gives

$$
\frac{2 G Q^{2}}{c^{4} r^{4}}=\frac{1}{B^{2}}\left(\frac{A^{\prime \prime}}{A}-\frac{A^{\prime}}{r A}-\frac{A^{\prime} B^{\prime}}{A B}-\frac{B^{\prime}}{r B}-\frac{1}{r^{2}}\right)+\frac{1}{r^{2}}
$$

The charge conservation Equation (10) is identically satisfied whereas (2) for energymomentum gives rise to the following surviving equation

$$
p^{\prime}=-\left(\rho c^{2}+p\right) \frac{A^{\prime}}{A}+\frac{Q Q^{\prime}}{4 \pi r^{4}}
$$

which is clearly contained in the field Equations (17) to (20).

We have three equations to determine five unknown functions $A, B, p, \rho$ and $\sigma$. Thus, we find a two degree of arbitrariness in the general relativistic problem of the 
electrically charged fluid ball, and to obtain a solution we can always choose one of these functions arbitrarily and correlate other to this by certain relation. The volume field surrounding the charged sphere is described by the Reissner-Nordstrom field

$$
\begin{gathered}
\mathrm{d} s^{2}=\left(1-\frac{2 G M}{c^{2} r}+\frac{G \epsilon^{2}}{c^{4} r^{2}}\right) c^{2} \mathrm{~d} t^{2}-\left(1-\frac{2 G M}{c^{2} r}+\frac{G \epsilon^{2}}{c^{4} r^{2}}\right)^{-1} \mathrm{~d} r^{2}-r^{2}\left(\mathrm{~d} \theta^{2}+\sin ^{2} \theta \mathrm{d} \phi^{2}\right) \\
F^{01}=-F^{10}=\frac{\epsilon}{r^{2}}
\end{gathered}
$$

where $M$ and $E$ are constants. We observe that whereas in Schwarzschilds field that total energy $M c^{2}$ is constant, in case of Reissner-Nordstrom field the total energy $\left(M c^{2}-\frac{\epsilon^{2}}{r}\right)$ increases as $r$ increases. At large distances the Reissner-Nordstron field approximates to Schwarzschilds field. The junction of interior and exterior field over the boundary $r=r_{\Sigma}$ of the sphere is governed by the junction conditions due to Darmois (1927), Viz. the continuity of the first and second fundamental forms across the boundary, which imply the continuity of $g_{\mu v}$ and the fluid pressure across the boundary. For the junction of electromagnetic field it is sufficient to consider the continuity of $F^{\mu v}$ (and not its first derivatives) across the boundary. In view of (16) and (23) the continuity of $F^{\mu \nu}$ gives

$$
\epsilon=Q_{\Sigma}
$$

Thus the constant $\epsilon$ measures the total charge contained within the ball.

Also we have

$$
\frac{1}{B^{2}}=1-\frac{2 G}{c^{2}} \frac{m(r)}{r}-\frac{2 G}{c^{4}} \frac{\mu(r)}{r}
$$

where

$$
m(r)=\int 4 \pi \rho r^{2} \mathrm{~d} r
$$

and

$$
\mu(r)=\int \frac{Q^{2}}{2 r^{2}} \mathrm{~d} r=\int 4 \pi r^{2} \frac{Q^{2}}{8 \pi r^{4}} \mathrm{~d} r
$$

The continuity of $g^{\mu \nu}$ over the boundary $r=r_{\Sigma}$ demands

$$
m\left(r_{\Sigma}\right)+\frac{\mu\left(r_{\Sigma}\right)}{c^{2}}=M-\frac{1}{2 c^{2}} \frac{\epsilon^{2}}{r_{\Sigma}}
$$

Thus, in general, the constant $M$ can not be identified with Euclidean mass of the sphere as against the case of uncharged sphere in which case $M=m\left(r_{\Sigma}\right)$. In view of (27) we have $M>m\left(r_{\Sigma}\right)$ we observe what the distribution of total energy within the charged sphere as measured by an electrically neutral test particle close to the boundary of the star is similar to that within a sphere of electrically neutral perfect fluid. Equation (27) can be rewritten as,

$$
M c^{2}=\int_{0}^{r_{\Sigma}} \rho c^{2} \cdot 4 \pi r^{2} \mathrm{~d} r+\int_{0}^{r_{\Sigma}} \frac{Q^{2}}{8 \pi r^{4}} \cdot 4 \pi r^{2} \mathrm{~d} r+\int_{\infty}^{r_{\Sigma}} \frac{Q^{2}}{8 \pi r^{4}} \cdot 4 \pi r^{2} \mathrm{~d} r
$$


Thus three physical quantities contribute to $M c^{2}$ viz. 1) the total matter energy within the ball distributed as if the geometry is Euclidean, 2) the total electromagnetic energy within the ball as if the geometry is Euclidean 3) the total electromagnetic energy distributed over the entire exterior space-time as if the geometry there too is euclidean.

The gravitational redshift of massive spherically symmetric ball is

$$
1+Z=g_{00}^{-\frac{1}{2}}
$$

which gives central $\left(Z_{0}\right)$ and surface $\left(Z_{\Sigma}\right)$ gravitational redshifts

$$
Z_{0}=\frac{C}{A}-1
$$

and

$$
Z_{\Sigma}=\left(1+\frac{r B^{\prime}}{B}\right)^{-1}-1
$$

\section{Gravational Binding Energy of a Charged Sphere}

In view of (11) the total non gravitational energy of a charged sphere is given

$$
E_{p}=\int_{0}^{r_{2}}\left(\rho c^{2}+\frac{Q^{2}}{8 \pi r^{4}}\right) \cdot 4 \pi r^{2} B \mathrm{~d} r
$$

Also the total energy of the ball measured by an electrically neutral test particle close to the boundary is given by

$$
E_{\Sigma}=M c^{2}-\frac{1}{2} \frac{\epsilon^{2}}{r_{\Sigma}}
$$

Clearly the difference $E_{p}-E_{\Sigma}$ is the expression for the gravitational energy of the charged fluid sphere as measured by an observer close to the boundary. The negative of this quantity is the gravitational binding energy of the system. In Newtonian limit we obtain from (24), (32) and (33)

$$
E_{p}-E_{\Sigma}=\int_{0}^{r_{\Sigma}} \frac{4 \pi G}{c^{4}} r\left(\rho c^{2}+\frac{Q^{2}}{8 \pi r^{4}}\right)\left[m(r) c^{2}+\mu(r)\right] . \mathrm{d} r
$$

For a particle at large distances from the object total energy $E_{\infty}$ approximates to $M c^{2}$. The difference $\frac{\epsilon^{2}}{2 r_{\Sigma}}$ between the two energy measurements is the classical expression for the energy of vacuum electrostatic field surrounding a sphere of charge $\epsilon$ and radius $r_{\Sigma}$. In the Reissner-Nordstrom field energy is distributed in the entire space time. As such the set of field Equations (17) to (19) can be solved under two given relations or assumptions. Physically speaking, one of them has to be the equation of state for fluid and another a law for the distribution of charge with the sphere. The non-singular solution due to Naduka [42] [43] and that due to Whitman and Burch [44] follow the charge distribution given by $\sigma=$ constant. $B^{-1}$. 


\section{Boundary Conditions for Well Behaved Solution}

For well behaved nature of the solution in isotropic coordinates, the following conditions should be satisfied:

1) The solution should be free from geometrical and physical singularities. Metric potentials A and B must be non-zero positive finite for free from geometrical singularities while central pressure, central density, should be positive and finite or $\rho_{0}>0$ and $p_{0}>0$ for free from physical singularities.

2) The solution should have maximum positive values of pressure and density at the center and monotonically decreasing towards the surface of fluid object i.e.

i) $\left(\frac{\mathrm{d} p}{\mathrm{~d} r}\right)_{0}=0$ and $\left(\frac{\mathrm{d}^{2} p}{\mathrm{~d} r^{2}}\right)_{0}<0$ such that the pressure gradient, $\frac{\mathrm{d} p}{\mathrm{~d} r}$ is negative for $0 \leq r \leq r_{\Sigma}$.

ii) $\left(\frac{\mathrm{d} \rho}{\mathrm{d} r}\right)_{0}=0$ and $\left(\frac{\mathrm{d}^{2} \rho}{\mathrm{d} r^{2}}\right)_{0}<0$ such that the density gradient, $\frac{\mathrm{d} \rho}{\mathrm{d} r}$ is negative for $0 \leq r \leq r_{\Sigma}$.

3) At boundary pressure, $p$ must vanish.

4) The pressure, $p$, and density $\rho$ should be positive.

5) Solution should have positive value of pressure-density ratio which must be less than 1 (weak energy condition) and less than $\frac{1}{3}$ (strong energy condition) throughout within the fluid object and monotonically decreasing as well (Pant and Negi [51]).

6) The casualty condition must be satisfied for this velocity of sound should be less than that of light throughout the model i.e. $0 \leq \sqrt{\frac{\mathrm{d} p}{c^{2} \mathrm{~d} \rho}}<1$. The velocity of sound should be monotonically decreasing towards the surface and increasing with the increase of density i.e. $\frac{\mathrm{d}}{\mathrm{d} r}\left(\frac{\mathrm{d} p}{\mathrm{~d} \rho}\right)<0$ or $\left(\frac{\mathrm{d}^{2} p}{\mathrm{~d} \rho^{2}}\right)>0$. In this context it is worth mentioning that the equation of state at ultra-high distribution has the property that the sound speed is decreasing outwards.

7) For realistic matter, the adiabatic index $\gamma>1$ i.e. $\frac{p}{\rho}<\frac{\mathrm{d} p}{\mathrm{~d} \rho}$, everywhere within the ball.

8) The red shift at the center $z_{0}$ and at the boundary should be positive, finite and monotonically decreasing in nature with the increase of $r$.

Under these conditions, we have to assume the one of the gravitational potential component in such a way that the field Equation (1) can be integrated and solution should be well behaved.

\section{New Class of Well Behaved Solution}

We present the following general analytic solution of the field Equations (17) to (20).

$$
A=b+d\left(1-a r^{2}\right)^{n+1}
$$




$$
B=\left(1-a r^{2}\right)^{n}
$$

The isotropic pressures, matter-energy density, charge, charge density and red shift of charged fluid ball are given by

$$
\begin{gathered}
\frac{8 \pi G}{c^{4}} p=\left\{\frac{-4 a(n+1) d}{\left(1-a r^{2}\right)^{n}\left[b+d\left(1-a r^{2}\right)^{n+1}\right]}+\frac{1+(2 n-1) a r^{2}-\left(1-a r^{2}\right)^{2 n+1}}{2 r^{2}\left(1-a r^{2}\right)^{2 n+1}}\right\} \\
\frac{8 \pi G}{c^{2}} \rho=\left\{\frac{\left(1-a r^{2}\right)^{2 n+1}-(10 n-1) a r^{2}-1}{2 r^{2}\left(1-a r^{2}\right)^{2 n+1}}\right\} \\
\frac{8 \pi \sqrt{G} \sigma}{c^{2}}=\frac{\sqrt{2}\left\{\left(1-a r^{2}\right)^{2 n+2}+\left(4 n^{2}-1\right) a^{2} r^{4}+2(n+1) a r^{2}-1\right\}}{c^{4} r^{4}}=\frac{\left(1-a r^{2}\right)^{2 n+1}+(2 n+1) a r^{2}-1}{r^{2}\left(1-a r^{2}\right)^{2 n+1}} \\
Z=\frac{(c-b)-d\left(1-a r^{2}\right)^{n-1}}{b-d\left(1-a r^{2}\right)^{n-1}}
\end{gathered}
$$

Here $a, b$, and $d$ are arbitrary constants.

In order to construct a new relativistic model, we assume $n=-2$ then we have

$$
\begin{gathered}
A=b+d\left(1-a r^{2}\right)^{-1} \\
B=\left(1-a r^{2}\right)^{-2} \\
\frac{8 \pi G}{c^{4}} p=\frac{a}{2}\left\{\frac{8 d\left(1-a r^{2}\right)^{3}}{b\left(1-a r^{2}\right)+d}-8+18 a r^{2}-16 a^{2} r^{4}+5 a^{3} r^{6}\right\} \\
\frac{8 \pi G}{c^{2}} \rho=\frac{a}{2}\left(24-66 a r^{2}+64 a^{2} r^{4}-21 a^{3} r^{6}\right) \\
\frac{2 G Q^{2}}{c^{4}}=a^{2} r^{6}\left(6-8 a r^{2}+3 a^{2} r^{4}\right) \\
\frac{8 \pi \sqrt{G} \sigma}{c^{2}}=a \frac{\left(1-a r^{2}\right)^{2}\left(36-64 a r^{2}+30 a^{2} r^{4}\right)}{\left(12-16 a r^{2}+6 a^{2} r^{4}\right)^{\frac{1}{2}}} \\
Z=\frac{(c-b)\left(1-a r^{2}\right)-d}{b\left(1-a r^{2}\right)+d}
\end{gathered}
$$

Here $a, b$, and $d$ are arbitrary constants.

The variation in pressure, density, charge, charge density and red shift with radial distance are given as 


$$
\begin{gathered}
\frac{8 \pi G}{c^{4}} p^{\prime}=-a^{2} r\left[\frac{8 d\left(1-a r^{2}\right)^{2}\left\{2 b\left(1-a r^{2}\right)+3 d\right\}}{\left\{b\left(1-a r^{2}\right)+d\right\}^{2}}-18+32 a r^{2}-15 a^{2} r^{4}\right] \\
\frac{8 \pi G}{c^{2}} \rho^{\prime}=-a^{2} r\left(66-128 a r^{2}+63 a^{2} r^{4}\right) \\
\frac{\sqrt{G} Q^{\prime}}{c^{2}}=\frac{a r^{2}\left(18-32 a r^{2}+15 a^{2} r^{4}\right)}{\left(12-16 a r^{2}+6 a^{2} r^{4}\right)^{\frac{1}{2}}} \\
Z^{\prime}=\frac{-2 c a d r}{\left\{b\left(1-a r^{2}\right)+d\right\}^{2}}
\end{gathered}
$$

\section{Properties of the Solution}

For real values of metric potentials $A$ and $B, a r^{2}<1 \forall r$. Figure 1 shows that the metric potentials $A$ and $B$ are positive at the center which are slightly and monotonically increasing with $r$ for suitable choice of constants $a, b$, and $d$. For the positive central value of $A(b+d)>0$,

The central value of $p, \rho$ and $\sigma$ are given as,

$$
\begin{gathered}
\frac{8 \pi G}{c^{4}} p_{0}=-\frac{(4 a b)}{(b+d)} \\
\frac{8 \pi G}{c^{2}} \rho_{0}=12 a
\end{gathered}
$$

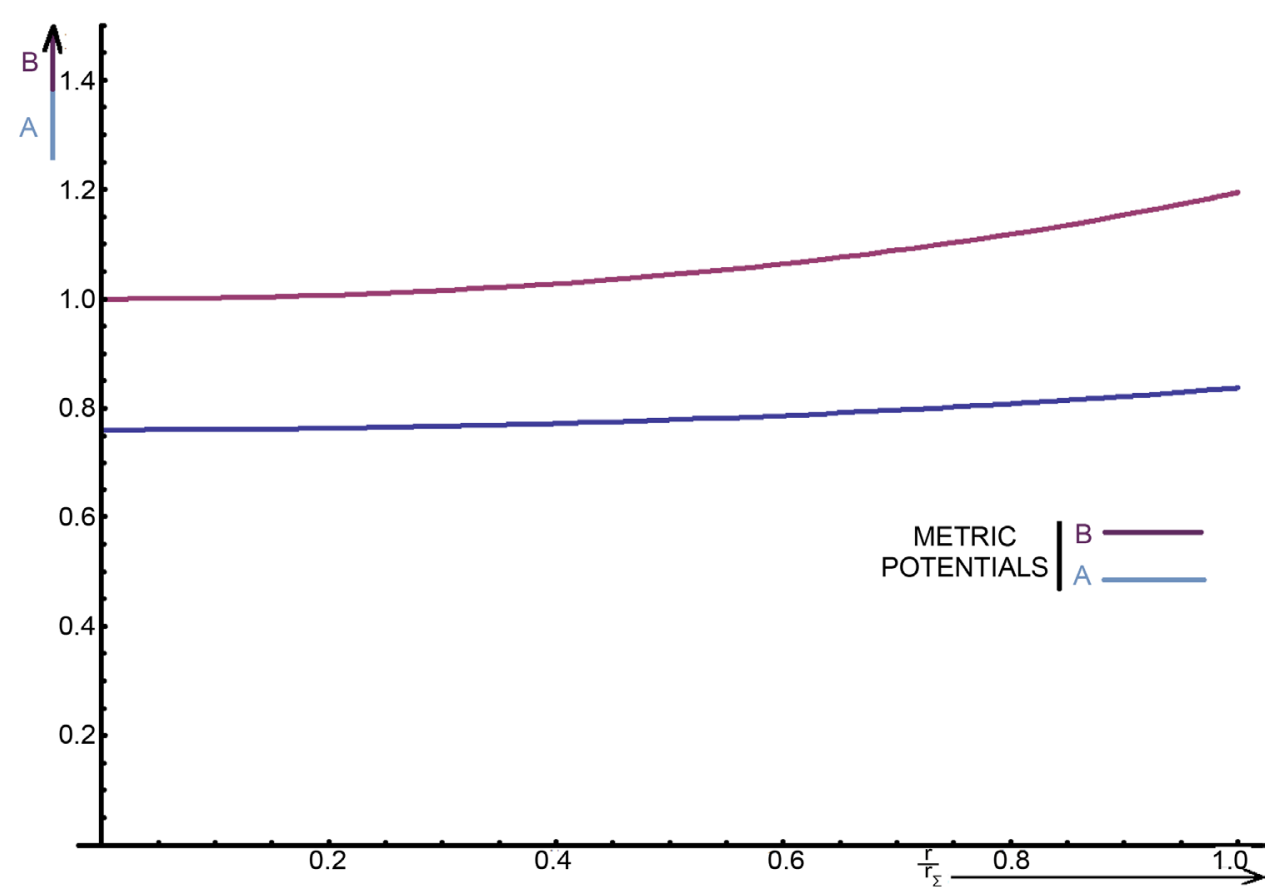

Figure 1. Variation of metric potentials with $\frac{r}{r_{\Sigma}}$. 


$$
\begin{gathered}
Q_{0}=0 \\
\frac{8 \pi \sqrt{G}}{c^{2}} \sigma_{0}=6 \sqrt{3} a \\
Z_{0}=\frac{c-b-d}{b+d}
\end{gathered}
$$

It is clear from Equation (53) to Equation (57) that for positive central values of physical quantities $p_{0}, \rho_{0}, Q_{0}, \sigma_{0}$ and $Z_{0}$ are positive if $a>0, b+d<c$ and $-1<\frac{b}{d}<0$. In view of Equations (49) and (50), the variation in the pressure and density with the distance from the center of fluid ball are identically zero at the center.

At the center of fluid ball the second order derivatives of pressure and density with respect to radial distance from the center of fluid ball are

$$
\frac{8 \pi G}{c^{4}}\left(p^{\prime \prime}\right)_{0}=2 a^{2} \frac{\left(9 b^{2}+10 b d-3 d^{2}\right)}{(b+d)^{2}}
$$

The pressure is maximum at the center if $\left(p^{\prime \prime}\right)_{0}<0$ i.e. $\frac{-5-\sqrt{52}}{9}<\frac{b}{d}<\frac{-5+\sqrt{52}}{9}$.

$$
\frac{8 \pi G}{c^{4}}\left(\rho^{\prime \prime}\right)_{0}=-66 a^{2}
$$

The density is maximum at the center for all constants as $\left(\rho^{\prime \prime}\right)_{0}<0$.

The central equation of state

$$
\left(\frac{p}{c^{2} \rho}\right)_{0}=\alpha=-\frac{b}{3(b+d)}
$$

$\alpha$ must satisfies the condition $0<\alpha \leq 1$ which demands $b<0$ and $-1<\frac{b}{d}<0$.

The central value of $\frac{\mathrm{d} p}{c^{2} \mathrm{~d} \rho}$ is given by

$$
\left(\frac{\mathrm{d} p}{c^{2} \mathrm{~d} \rho}\right)_{0}=-\frac{\left(9 b^{2}+10 b d-3 d^{2}\right)}{33(b+d)^{2}}
$$

The causality condition at the center $0<\left(\frac{\mathrm{d} p}{c^{2} \mathrm{~d} \rho}\right)_{0} \leq 1$ gives $\frac{-5-\sqrt{52}}{9}<\frac{b}{d}<\frac{-5+\sqrt{52}}{9}$.

It is found that $\rho$ and $\sigma$ fall monotonically from their maximum positive values at the center up to non negative values at the boundary (Figure 2) and $p$ falls monotonically from it's maximum positive values at the center up to zero value at the boundary while charge increases from zero at the center to maximum positive value at the boundary (Figure 3) for different values of the parameters $a, b, d$ satisfying

$$
a>0, \quad b<0, \quad d>0, \quad c>b+d>0 \quad \text { and } \quad-1<\frac{b}{d}<0
$$




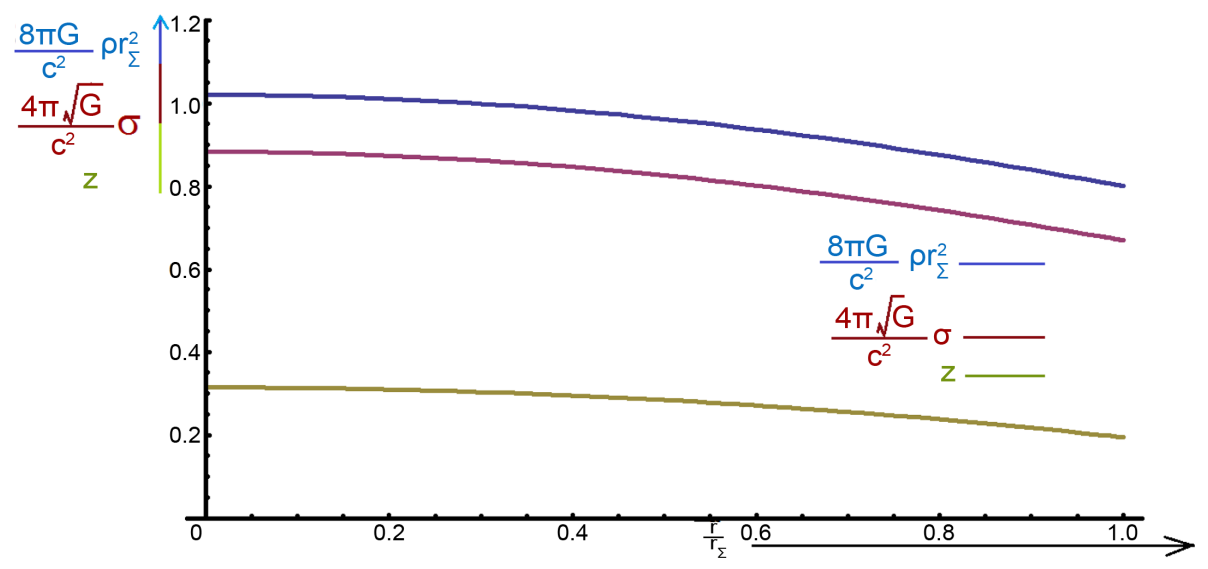

Figure 2. Variation of energy density, charge density and red shift with $\frac{r}{r_{\Sigma}}$.

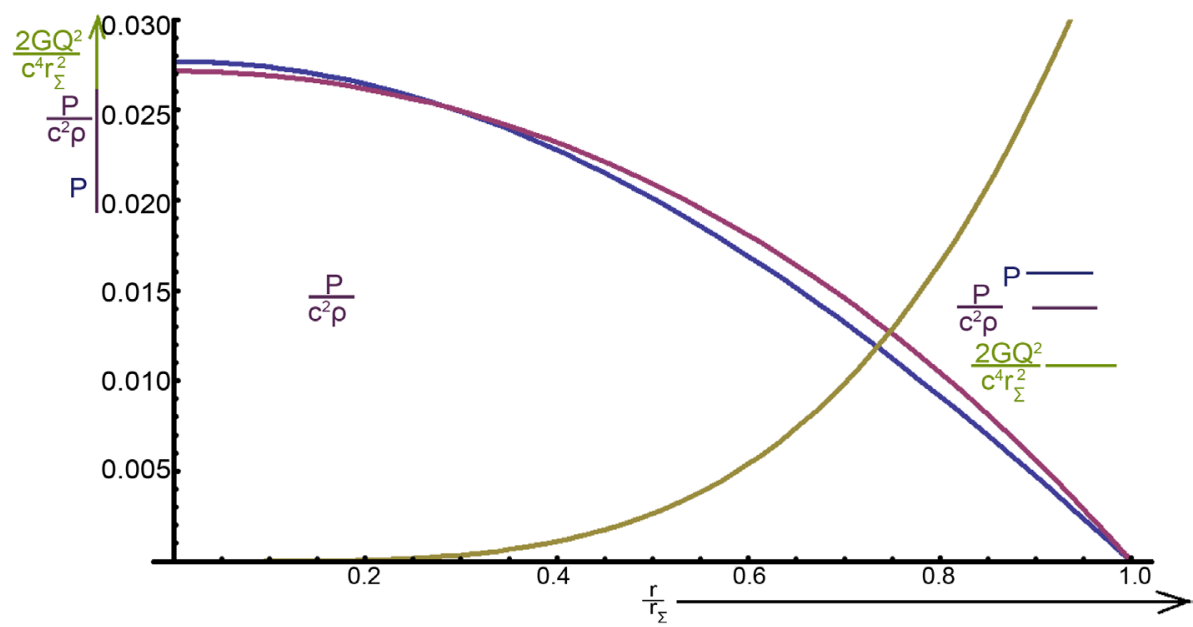

Figure 3. Variation of pressure, pressure density ratio and charge with $\frac{r}{r_{\Sigma}}$.

Figure 4 shows that speed of sound is less than speed of light i.e. $0<\frac{\mathrm{d} p}{c^{2} \mathrm{~d} \rho} \leq 1$ and the ratio of speeds of sound and light $\sqrt{\frac{\mathrm{d} p}{c^{2} \mathrm{~d} \rho}}$ falls monotonically from center to the boundary of the fluid ball.

\section{Matching Conditions of Boundary}

The solution so obtained are to be matched over the pressure free boundary of fluid sphere smoothly with the Reissner-Nordstrom metric:

$$
\mathrm{d} s^{2}=\left(1-\frac{2 G M}{c^{2} r}+\frac{G \epsilon^{2}}{c^{4} r^{2}}\right) c^{2} \mathrm{~d} t^{2}-\left(1-\frac{2 G M}{c^{2} r}+\frac{G \epsilon^{2}}{c^{4} r^{2}}\right)^{-1} \mathrm{~d} r^{2}-r^{2}\left(\mathrm{~d} \theta^{2}+\sin ^{2} \theta \mathrm{d} \phi^{2}\right)
$$

which requires the continuity of $A^{2}$ and $B^{2}$ across the boundary $r=r_{\Sigma}$ and $(p)_{r_{\Sigma}}=0 ; Q_{r_{\Sigma}}=\epsilon$. Thus 


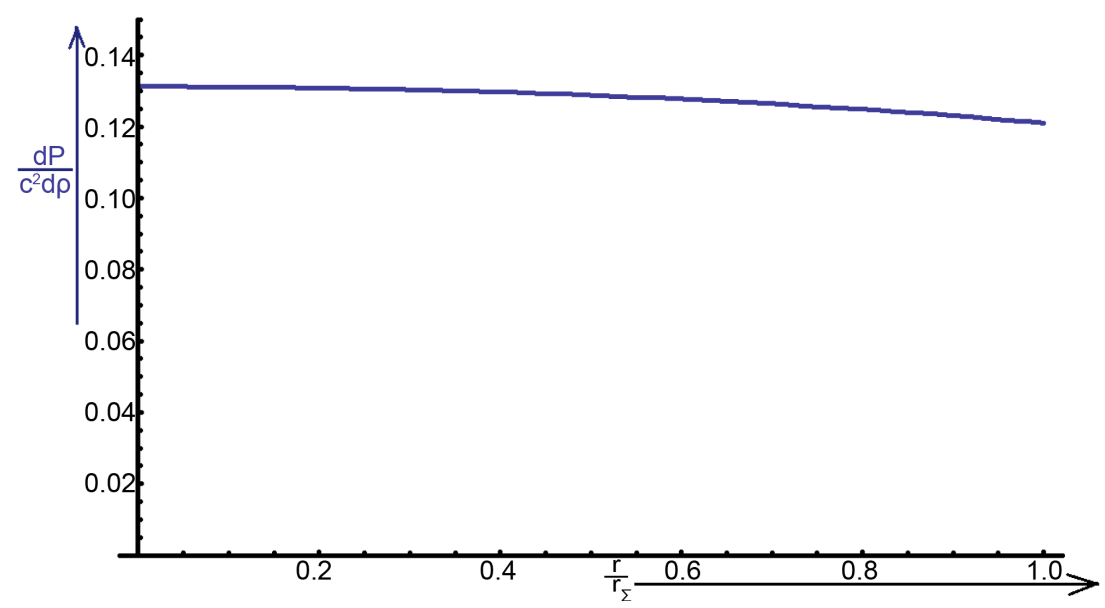

Figure 4. Variation of adiabatic velocity of sound with $\frac{r}{r_{\Sigma}}$.

$$
\begin{gathered}
a=\frac{1}{r_{\Sigma}^{2}}\left(1-X^{\frac{1}{4}}\right) ; \quad 0<X<1 \\
b=\frac{\left(3 X^{\frac{3}{4}}-X^{\frac{1}{2}}-X^{\frac{1}{4}}-1\right) c}{8 X^{\frac{1}{4}}} \\
d=\frac{c}{8}\left(5 X^{\frac{3}{4}}+X^{\frac{1}{2}}+X^{\frac{1}{4}}+1\right)
\end{gathered}
$$

where $X=1-2 S_{p}+\frac{G Q_{\Sigma}^{2}}{c^{4} r_{\Sigma}^{2}} ; S_{p}=\frac{G M}{c^{2} r_{\Sigma}}$, Schwarzchild parameter.

\section{Tables of Numerical Values of Physical Quantities and Their Graphs}

In view of Equations (64) to (66) the values of $X, b, d$ and $\frac{b}{d}$ are $0.701,-0.062 c$, $0.0823 c$ and -0.0753 respectively and the value of $a r_{\Sigma}^{2}=0.085$ for $S_{p}=0.16$ and surface density $2 \times 10^{14} \mathrm{gmcm}^{-3}$. For better illustration of our physically accepted solution, the relevant physical quantities are presented by means of Table 1, Table 2 and Figures 1-4 for these constants.

In order to construct a super dense star model, we prescribe the surface density of the star as $2 \times 10^{17} \mathrm{kgm}^{-3}$ and the values of constants $a, b$ and $d$ can be evaluated for different values of $S_{p}$ for describing the well behaved solutions. Thus a compact star model can be constructed by finding mass and radius corresponding to assumed surface density. The variation in the mass and radius with schwarzschild parameters for our model of compact star is tabulated in Table 3 and the variation of surface charge, Surface Density, Schwarzschild Parameter and $X$ with $a r_{\Sigma}^{2}$ showing different models is shown in Figure 5. 
Table 1. $\frac{A}{c}, B, \rho, p$, and $\frac{p}{c^{2} \rho}$ for $a r_{\Sigma}^{2}=0.085$

\begin{tabular}{ccccccc}
\hline$S . N$. & $\frac{r}{r_{\Sigma}}$ & $\frac{A}{c}$ & $B$ & $\frac{8 \pi}{c^{2}} G \rho r_{\Sigma}^{2}$ & $\frac{8 \pi}{c^{4}} G p r_{\Sigma}^{2}$ & $\frac{p}{c^{2} \rho}$ \\
\hline 1 & 0.0 & 0.76085 & 1.00000 & 1.02000 & 0.02768 & 0.02715 \\
2 & 0.1 & 0.76155 & 1.00170 & 1.01762 & 0.02737 & 0.02690 \\
3 & 0.2 & 0.76365 & 1.00683 & 1.01048 & 0.02644 & 0.02617 \\
4 & 0.3 & 0.76719 & 1.01547 & 0.99862 & 0.02490 & 0.02493 \\
5 & 0.4 & 0.77219 & 1.02776 & 0.98210 & 0.02277 & 0.02319 \\
6 & 0.5 & 0.77871 & 1.04389 & 0.96099 & 0.02009 & 0.02091 \\
7 & 0.6 & 0.78682 & 1.06413 & 0.93541 & 0.01690 & 0.01806 \\
8 & 0.7 & 0.79661 & 1.08881 & 0.90546 & 0.01323 & 0.01462 \\
9 & 0.8 & 0.80818 & 1.11837 & 0.87129 & 0.00915 & 0.01050 \\
10 & 0.9 & 0.82169 & 1.15335 & 0.83303 & 0.00471 & 0.00565 \\
11 & 1.0 & 0.83729 & 1.19442 & 0.79085 & 0.00000 & 0.00000 \\
\hline
\end{tabular}

Table 2. $\frac{\mathrm{d} p}{c^{2} \mathrm{~d} \rho}, \frac{\rho \mathrm{d} p}{p \mathrm{~d} \rho}, \sigma, Q$, and $Z$ for $a r_{\Sigma}^{2}=0.218$.

\begin{tabular}{ccccccc}
\hline$S . N$. & $\frac{r}{r_{\Sigma}}$ & $\frac{1}{c^{2}} \frac{\mathrm{d} p}{\mathrm{~d} \rho}$ & $\frac{\rho}{p} \frac{\mathrm{d} p}{\mathrm{~d} \rho}$ & $\frac{8 \pi \sqrt{G} \sigma}{c^{2}}$ & $\frac{G Q^{2}}{c^{4} r^{2}}$ & $Z$ \\
\hline 1 & 0.0 & 0.13119 & 4.83330 & 0.88334 & 0.00000 & 0.31432 \\
2 & 0.1 & 0.13099 & 4.86945 & 0.88107 & 0.00021 & 0.31311 \\
3 & 0.2 & 0.13037 & 4.98243 & 0.87412 & 0.00085 & 0.30948 \\
4 & 0.3 & 0.12935 & 5.18736 & 0.86295 & 0.00188 & 0.30345 \\
5 & 0.4 & 0.12791 & 5.51540 & 0.84794 & 0.00331 & 0.29501 \\
6 & 0.5 & 0.12605 & 6.02752 & 0.82972 & 0.00512 & 0.28416 \\
7 & 0.6 & 0.12376 & 6.84985 & 0.80905 & 0.00728 & 0.27093 \\
8 & 0.7 & 0.12104 & 8.28097 & 0.78683 & 0.00979 & 0.25532 \\
9 & 0.8 & 0.11788 & 11.22241 & 0.76402 & 0.01265 & 0.23733 \\
10 & 0.9 & 0.11427 & 20.20590 & 0.74168 & 0.01582 & 0.21700 \\
11 & 1.0 & 0.11020 & $\infty$ & 0.72088 & 0.01943 & 0.19433 \\
\hline
\end{tabular}

\section{Conclusion}

We have given a new solution for spherically symmetric isotropic charged fluid ball. It has been observed that the physical parameters pressure, density, adiabatic speed of sound and redshift are positive at the centre and within the limit of realistic state equation and monotonically decreasing and the causality condition is obeyed throughout the fluid ball. The charge and electric field intensity are zero at the center and monotonincally increasing towards the intervening surface. Thus, the solution is well behaved for all values of Schwarzschild parameter $S_{p}$ within the charged fluid ball. Our solution is useful to construct the models of compact star like Strange star family, 
Table 3. Variation in $a r_{\Sigma}^{2}, \frac{G Q^{2}}{c^{4} r_{\Sigma}^{2}}, S_{p}, X, \frac{8 \pi}{c^{2}} G \rho r_{\Sigma}^{2}, r_{\Sigma}\left(\right.$ inKm) and $\frac{M}{M_{\odot}}$ with Schwarzschild parameter $S_{p}$.

\begin{tabular}{cccccccc}
\hline$S . N$. & $a r_{\Sigma}^{2}$ & $\frac{G Q^{2}}{c^{4} r_{\Sigma}^{2}}$ & $S_{p}$ & $X$ & $\frac{8 \pi}{c^{2}} G \rho r_{\Sigma}^{2}$ & $r_{\Sigma}(\mathrm{inKm})$ & $\frac{M}{M_{\odot}}$ \\
\hline 1 & 0.080 & 0.017 & 0.150 & 0.7164 & 0.765 & 14.330 & 1.451 \\
2 & 0.086 & 0.019 & 0.158 & 0.6978 & 0.810 & 14.750 & 1.573 \\
3 & 0.089 & 0.021 & 0.167 & 0.6887 & 0.831 & 14.940 & 1.684 \\
4 & 0.092 & 0.022 & 0.171 & 0.6797 & 0.851 & 15.118 & 1.745 \\
5 & 0.096 & 0.024 & 0.178 & 0.6678 & 0.873 & 15.312 & 1.840 \\
6 & 0.101 & 0.026 & 0.186 & 0.6531 & 0.904 & 15.582 & 1.956 \\
7 & 0.106 & 0.029 & 0.195 & 0.6387 & 0.936 & 15.855 & 2.087 \\
8 & 0.110 & 0.031 & 0.202 & 0.6274 & 0.961 & 16.065 & 2.190 \\
9 & 0.115 & 0.034 & 0.209 & 0.6134 & 0.988 & 16.289 & 2.290 \\
10 & 0.120 & 0.036 & 0.218 & 0.5996 & 1.017 & 16.527 & 2.432 \\
11 & 0.126 & 0.040 & 0.228 & 0.5835 & 1.048 & 16.777 & 2.582 \\
12 & 0.130 & 0.042 & 0.235 & 0.5728 & 1.070 & 16.952 & 2.689 \\
13 & 0.134 & 0.045 & 0.242 & 0.5624 & 1.091 & 17.118 & 2.796 \\
14 & 0.140 & 0.049 & 0.251 & 0.5470 & 1.119 & 17.336 & 2.937 \\
15 & 0.145 & 0.052 & 0.259 & 0.5344 & 1.141 & 17.505 & 3.060 \\
\hline
\end{tabular}

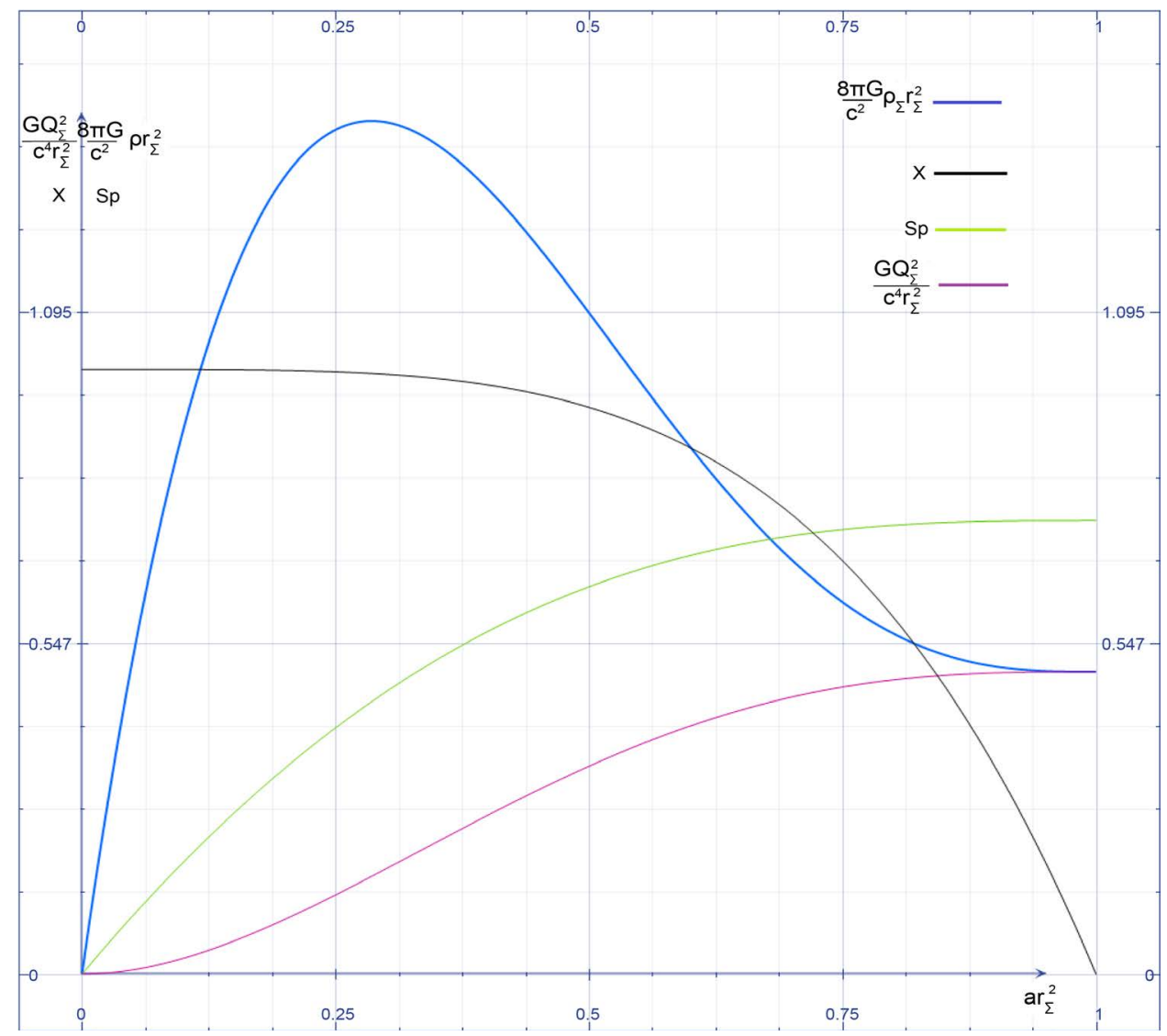

Figure 5. Variation of surface charge, surface density, Schwarzschild parameter and $X$ with $a r_{\Sigma}^{2}$ showing different models. 
Neutron star and many more. We have discussed a model of massive neutron star having mass $1.58 \mathrm{M} \odot$ and radius $14.66 \mathrm{~km}$ with surface density $2 \times 10^{17} \mathrm{Kgm}^{-3}$ and central density $2.6 \times 10^{17} \mathrm{kgm}^{-3}$. The central pressure of neutron star is $6.2 \times 10^{32} \mathrm{~N} / \mathrm{m}^{2}$ while the surface pressure of the star is zero. The electric field intensity at the center is zero and at the surface it comes $2.37 \times 10^{31} \mathrm{C}^{2} \mathrm{~m}^{-2}$. Table 3 shows that we can construct different models for neutron star having mass lies between $1.45 \mathrm{M} \odot$ and $3.06 \mathrm{M} \odot$ for different values of $S_{p}$ ranging from 0.15 to 0.259 . The solution reduces to Schwarzschild interior solution for $n=-1 / 2$ for electrically neutral fluid ball.

\section{References}

[1] Schwarzschild, K. (1916) On the Gravitational Field of a Mass Point According to Einstein's Theory. Sitzungsberichte der koniglich Preussischen Akademie der Wissenschaften Berlin (Mathematical Physics), S42, 189-196.

[2] Bonnor, W.B. (1960) The Mass of a Static Charged Sphere. Zeitschrift für Physik, 160, 59.

[3] Bonnor, W.B. (1965) The Equilibrium of a Charged Sphere. Monthly Notices of the Royal Astronomical Society, 129, 443. https://doi.org/10.1093/mnras/129.6.443

[4] Oppenheimer, J.R. and Snyder, H. (1939) On Continued Gravitational Contraction. Physical Review, 56, 455. https://doi.org/10.1103/PhysRev.56.455

[5] Shvartsman, V.F. (1971) The Electric Charge of Stars. Zhurnal Eksperimental noi i Teoreticheskoi Fiziki, 33, 475-648.

[6] Buchdahl, H.A. (1959) General Relativistic Fluid Spheres. Physical Review, 116, 1027. https://doi.org/10.1103/PhysRev.116.1027

[7] Delgaty, M.S.R. and Lake, K. (1998) Physical Acceptability of Isolated, Static, Spherically Symmetric, Perfect Fluid Solutions of Einstein's Equations. Computer Physics Communications, 115, 395-415. https://doi.org/10.1016/S0010-4655(98)00130-1

[8] Adler, R.J. (1974) A Fluid Sphere in General Relativity. Journal of Mathematical Physics, 15, 727. https://doi.org/10.1063/1.1666717

[9] Heintzmann, H. (1969) New Exact Static Solutions of Einstein's Field Equations. Zeitschrift für Physik, 228, 489-493.

[10] Tolman, R.C. (1939) Static Solutions of Einstein's Field Equations for Spheres of Fluid. Physical Review, 55, 364. https://doi.org/10.1103/PhysRev.55.364

[11] Finch, N.R. and Skea, J.E.F. (1989) A Realistic Stellar Model Based on an Ansatz of Duorah and Ray. Classical and Quantum Gravity, 6, 467-476. https://doi.org/10.1088/0264-9381/6/4/007

[12] Patwardhan, G.K. and Vaidya, P.C. (1943) Relativistic Distributions of Matter of Radial Symmetry. Journal of the University of Bombay, 12, 23-26.

[13] Mehra, A.L. (1966) Radially Symmetric Distribution of Matter. Journal of the Australian Mathematical Society, 6, 153-156. https://doi.org/10.1017/S1446788700004730

[14] Kuchowicz, B. (1968) General Relativistic Fluid Spheres. II. Solutions of the Equation for e/sup-lambda/. Acta Physica Polonica, 34, 131-140.

[15] Matese, J.J. and Whitman, P.G. (1980) New Method for Extracting Static Equilibrium Configurations in General Relativity. Physical Review D, 22, 1270-1275.

https://doi.org/10.1103/PhysRevD.22.1270

[16] Durgapal, M.C. (1982) A Class of New Exact Solutions in General Relativity. Journal of Physics A: Mathematical and General, 15, 2637-2644. 
https://doi.org/10.1088/0305-4470/15/8/039

[17] Nariai, S. (1950) On Some Static Solutions of Einstein's Gravitational Field Equations in a Spherically Symmetric Case. The Science Reports of the Tohoku University, 34, 160.

[18] Goldman, S.P. (1978) Physical Solutions to General-Relativistic Fluid Sphere. Astrophysical Journal, 226, 1079-1086. https://doi.org/10.1086/156684

[19] Ivanov, B.V. (2012) Collapsing Shear-Free Perfect Fluid Spheres with Heat Flow. General Relativity and Gravitation, 44, 1835-1855. https://doi.org/10.1007/s10714-012-1370-3

[20] Ivanov, B.V. (2010) Evolving Spheres of Shear-Free Anisotropic Fluid. International Journal of Modern Physics A, 25, 3975-3991. https://doi.org/10.1142/S0217751X10050202

[21] Ivanov, B.V. (2011) Self-Gravitating Spheres of Anisotropic Fluid in Geodesic Flow. International Journal of Modern Physics D, 20, 319-334. https://doi.org/10.1142/S0218271811018858

[22] Ivanov, B.V. (2016) A Different Approach to Anisotropic Spherical Collapse with Shear and Heat Radiation. International Journal of Modern Physics D, 25, Article ID: 1650049. https://doi.org/10.1142/s0218271816500498

[23] Ivanov, B.V. (2016) All Solutions for Geodesic Anisotropic Spherical Collapse with Shear and Heat Radiation. Astrophysics and Space Science, 361, 18. https://doi.org/10.1007/s10509-015-2603-1

[24] Pant, N. (2011) Some New Exact Solutions with Finite Central Parameters and Uniform Radial Motion of Sound. Astrophysics and Space Science, 331, 633-644. https://doi.org/10.1007/s10509-010-0453-4

[25] Maurya, S.K. and Gupta, K. (2013) Charged Fluid to Anisotropic Fluid Distribution in General Relativity. Astrophysics and Space Science, 344, 243-251. https://doi.org/10.1007/s10509-012-1302-4

[26] Pant, N., Fuloria, P. and Tewari, B.C. (2012) A New Well Behaved Exact Solution in General Relativity for Perfect Fluid. Astrophysics and Space Science, 340, 407-412. https://doi.org/10.1007/s10509-012-1068-8

[27] Pant, N., Fuloria, P. and Pradhan, N. (2014) An Exact Solution of Perfect Fluid in Isotropic Coordinates, Compatible with Relativistic Modeling of Star. International Journal of Theoretical Physics, 53, 993-1002. https://doi.org/10.1007/s10773-013-1892-9

[28] Pant, D.N. and Sah, A. (1982) Class of Solutions of Einstein's Field Equations for Static Fluid Spheres. Physical Review D, 26, 1254-1261.

https://doi.org/10.1103/PhysRevD.26.1254

[29] Pant, D.N. and Sah, A. (1985) Massive Fluid Spheres in General Relativity. Physical Review D, 32, 1358-1363. https://doi.org/10.1103/PhysRevD.32.1358

[30] Tewari, B.C., Charan, K. and Chandra, P. (2015) A Well Behaved Exact Solution for Spherically Symmetric Perfect Fluid Ball. Journal of Ramanujan Society of Mathematics and Mathematical Sciences, 4, 77-86.

[31] Sah, A., Chandra, P. and Charan, K. (2016) Spherically Symmetric Anisotropic Perfect Fluid Ball in General Relativity. South East Asian Journal of Mathematics and Mathematical Science, 12, 81-92.

[32] Herrera, L. and Santos, N.O. (1997) Local Anisotropy in Self-Gravitating Systems. Physics Reports, 286, 53-130. https://doi.org/10.1016/S0370-1573(96)00042-7

[33] Herrera, L., Santos, N.O. and Wang, A. (2008) Shearing Expansion-Free Spherical Anisotropic Fluid Evolution. Physical Review D, 78, Article ID: 084026.

https://doi.org/10.1103/PhysRevD.78.084026 
[34] Herrera, L., Ospino, J. and Perisco, A.D. (2008) All Static Spherically Symmetric Anisotropic Solutions of Einstein's Equations. Physical Review D, 77, Article ID: 027502. https://doi.org/10.1103/PhysRevD.77.027502

[35] Herrera, L., Ospino, J., Perisco, A.D., Fuenmayor, E. and Triconis, O. (2009) Structure and Evolution of Self-Gravitating Objects and the Orthogonal Splitting of the Riemann Tensor. Physical Review D, 79, Article ID: 064025. https://doi.org/10.1103/physrevd.79.064025

[36] Herrera, L. Parisco, A.D., Hernandez, P. and Santos, N.O. (2004) Dynamics of Dissipative Gravitational Collapse. Physical Review D, 70, Article ID: 084004. https://doi.org/10.1103/physrevd.70.084004

[37] Herrera, L., Parisco, A.D., Hernandez, P. and Santos, N.O. (1998) On the Role of Density Inhomogeneity and Local Anisotropy in the Fate of Spherical Collapse. Physics Letters A, 237, 113-118. https://doi.org/10.1016/S0375-9601(97)00874-8

[38] Tewari, B.C. and Charan, K. (2015) Horizon Free Eternally Collapsing Anisotropic Radiating Star. Astrophysics and Space Science, 357, 107. https://doi.org/10.1007/s10509-015-2335-2

[39] Tewari, B.C. and Charan, K. (2014) Radiating Star, Shear-Free Gravitational Collapse without Horizon. Astrophysics and Space Science, 351, 613-617. https://doi.org/10.1007/s10509-014-1851-9

[40] Tewari, B.C., Charan, K. and Rani, J. (2016) Spherical Gravitational Collapse of Anisotropic Radiating Fluid Sphere. International Journal of Astronomy and Astrophysics, 6, 155-165. https://doi.org/10.4236/ijaa.2016.62013

[41] Tewari, B.C. (2013) Collapsing Shear-Free Radiating Fluid Spheres. General Relativity and Gravitation, 45, 1547-1558. https://doi.org/10.1007/s10714-013-1545-6

[42] Nduka, A. (1976) Charged Fluid Sphere in General Relativity. General Relativity and Gravitation, 7, 493-499. https://doi.org/10.1007/BF00766408

[43] Nduka, A. (1977) Some Exact Solutions Charged General Relativistic Fluid Sphere. Acta Physica Polonica B, 8, 75-79.

[44] Whitman, P.G. and Burch, R.C. (1982) Charged Spheres in General Relativity. Physical Review D, 24, 2049-2055. https://doi.org/10.1103/PhysRevD.24.2049

[45] Tikekar, R. (1984) Spherical Charged Fluid Distributions in General Relativity. Journal of Mathematical Physics, 25, 1481-1483. https://doi.org/10.1063/1.526318

[46] Ivanov, B.V. (2002) Static Charged Perfect Fluid Spheres in General Relativity. Physical Review D, 65, Article ID: 104001. https://doi.org/10.1103/physrevd.65.104001

[47] Ray, S., Espindola, A., Malheiro, M., Lemos, J. and Zanchin, V. (2003) Electrically Charged Compact Stars and Formation of Charged Black Hole. Physical Review D, 68, Article ID: 084004. https://doi.org/10.1103/physrevd.68.084004

[48] Stettner, R. (1973) On the Stability of Homogeneous, Spherically Symmetric, Charged Fluid in Relativity. Annals of Physics, 80, 212-227. https://doi.org/10.1016/0003-4916(73)90325-4

[49] Krori, K.D. and Barua, J. (1975) A Singularity Free Solution for a Charged Fluid Sphere in General Relativity. Journal of Physics A: Mathematical and General, 8, 508-511. https://doi.org/10.1088/0305-4470/8/4/012

[50] Ray, S. and Das, B. (2004) Tolman-Bayin Type Static Charged Fluid Spheres in General Relativity. Monthly Notices of the Royal Astronomical Society, 349, 1331-1334. https://doi.org/10.1111/j.1365-2966.2004.07602.x

[51] Pant, N. and Negi, P.S. (2012) Variety of Well Behaved Exact Solutions of Einstein-Maxwell Field Equations: An Application to Strange Quark Stars, Neutron Stars and Pulsars. Astro- 
physics and Space Science, 338, 163-169. https://doi.org/10.1007/s10509-011-0919-z

[52] Florides, P.S. (1983) The Complete Field of Charged Perfect Fluid Spheres and Other Static Spherically Symmetric Charged Distributions. Journal of Physics A: Mathematical and General, 16, 1419-1433. https://doi.org/10.1088/0305-4470/16/7/018

[53] Dionysiou, D.D. (1982) Equilibrium of a Static Charged Perfect Fluid Sphere. Astrophysics and Space Science, 85, 331-343. https://doi.org/10.1007/BF00653455

[54] Pant, N., Tewari, B. and Fuloria, P. (2011) Well Behaved Class of Exact Solutions of Einstein-Maxwell Field Equations in General Relativity. Astrophysics and Space Science, 2, 1538-1543.

[55] Pant, N., Mehta, R.N. and Pant, M. (2011) Well Behaved Class of Charge Analogue of Heintzmann's Relativistic Exact Solution. Astrophysics and Space Science, 332, 473-479. https://doi.org/10.1007/s10509-010-0509-5

[56] Pant, M.J. and Tewari, B.C. (2011) Well Behaved Class of Charge Analogue of Adler's Relativistic Exact Solution. Journal of Modern Physics, 2, 481-487. https://doi.org/10.4236/jmp.2011.26058

[57] Fuloria, P., Tewari, B.C. and Joshi, B.C. (2011) Well Behaved Class of Charge Analogue of Durgapal's Reltivistic Exact Solution. Journal of Modern Physics, 2, 1156-1160. https://doi.org/10.4236/jmp.2011.210143

[58] Gupta, Y.K. and Maurya, S.K. (2010) A Class of Charge Analogues of Durgapal and Fuloria Superdense Star. Astrophysics and Space Science, 331, 135-144. https://doi.org/10.1007/s10509-010-0445-4

[59] Bijalwan, N. and Gupta, Y.K. (2008) Nonsingular Charged Analogues of Schwarzschild's Interior Solution. Astrophysics and Space Science, 317, 251-260. https://doi.org/10.1007/s10509-008-9887-3

[60] Gupta, Y.K. and Kumar, M. (2005) A Superdense Star Model as Charged Analogue of Schwarzschild's Interior Solution. General Relativity and Gravitation, 37, 575-583. https://doi.org/10.1007/s10714-005-0043-X

[61] Sah, A. and Chandra, P. (2016) Spherical Anisotropic Fluid Distribution in General Relativity. World Journal of Mechanics, 6, 487-504. http://dx.doi.org/10.4236/wjm.2016.612034

\section{Scientific Research Publishing}

\section{Submit or recommend next manuscript to SCIRP and we will provide best service for you:}

Accepting pre-submission inquiries through Email, Facebook, LinkedIn, Twitter, etc. A wide selection of journals (inclusive of 9 subjects, more than 200 journals)

Providing 24-hour high-quality service

User-friendly online submission system

Fair and swift peer-review system

Efficient typesetting and proofreading procedure

Display of the result of downloads and visits, as well as the number of cited articles

Maximum dissemination of your research work

Submit your manuscript at: http://papersubmission.scirp.org/

Or contactijaa@scirp.org 